\title{
INTERACTION OF HIGH-POWER TERAHERTZ RADIATION WITH METALLIC FILMS
}

\author{
O. V. Chefonov $^{1}$, A. V. Ovchinnikov ${ }^{1}$, S. I. Ashitkov ${ }^{1}$, S. A. Evlashin ${ }^{2}$, P. S. Kondratenko ${ }^{3}$, \\ M. B. Agranat $^{1}$, V. E. Fortov ${ }^{1}$ \\ ${ }^{1}$ Joint Institute for High Temperatures of the Russian Academy of Sciences, Moscow, Russia, oleg.chefonov@gmail.com \\ ${ }^{2}$ Center for Design, Manufacturing and Materials, Skolkovo Institute of Science and Technology, Moscow, Russia \\ ${ }^{3}$ Nuclear Safety Institute of the Russian Academy of Sciences, Moscow, Russia
}

We report on the experimental observation of high-power terahertz (THz) radiation-induced damage in thin aluminum and nickel films with a thickness less than a terahertz skin-depth. The damage threshold fluence of thin metal films is evaluated for single pulse experiments.

The experiments were performed using a terawatt femtosecond Cr:forsterite laser system, that delivers 100 -fs pulses with a bandwidth of $25 \mathrm{~nm}$ at a central wavelength of $1240 \mathrm{~nm}$ with energy above $80 \mathrm{~mJ}$ (energy stability less than $10 \%$ ) at $10 \mathrm{~Hz}$ repetition rate [1]. THz pulses in the range of $1-3 \mathrm{THz}$ were generated by optical rectification of femtosecond laser pulses in organic nonlinear crystal DSTMS (4-N,Ndimethylamino-4-N-methyl-stilbazolium 2,4,6trimethylbenzene-sulfonate) of $8 \mathrm{~mm}$ in diameter [2, 3]. $\mathrm{THz}$ low pass filter was used for cutting wavelengths below $34 \mu \mathrm{m}$, including the pumping radiation at a wavelength of $1240 \mathrm{~nm}$ and its second harmonic at $620 \mathrm{~nm}$ generated in nonlinear crystal during optical rectification process. The attenuation coefficient of the THz low pass filter at wavelengths of $1240 \mathrm{~nm}$ and $620 \mathrm{~nm}$ was more than $10^{8}$. A telescope 6:1 consisted of two off-axis parabolic mirrors with a reflected focus length of 25.4 and $152.4 \mathrm{~mm}$ was used to compensate $\mathrm{THz}$ beam divergence. The collimated $\mathrm{THz}$ beam traveling from the telescope was focused onto a sample using an $90^{\circ}$ off-axis parabolic mirror with a reflected focus length of $50.8 \mathrm{~mm}$ and a diameter of $50.8 \mathrm{~mm}$ to a diffraction-limited spot size. Terahertz pulse energy was measured by means of a calibrated optoacoustic detector (Golay cell) and was up to $\sim 110$ $\mu \mathrm{J}$ at the samples surface.

The samples were a $20-\mathrm{nm} \mathrm{Al}$ and $\mathrm{Ni}$ films deposited on a glass substrate of $180 \mu \mathrm{m}$ in thickness using magnetron sputtering of a high-clean metal target (purity of $99.99 \%$ ) at a rate of $1-1.91 \mathrm{~nm} / \mathrm{s}$ in an argon atmosphere (purity of $99.999 \%)$. The scatter in films thickness were about 1-3 nm from the specified value.

Scanning electron microscope (SEM) images of thin metal film damage patterns at different fluences $F$ of a single THz pulse are presented in Fig. 1 and Fig. 2.

The single-pulse damage threshold of the thin film was experimentally determined using a standard technique, where the squared radius $\left(r_{\mathrm{D}}[\mu \mathrm{m}]\right)$ of observed damaged regions is plotted versus the logarithm of the numerical value of the energy of incident $\mathrm{THz}$ pulses $W_{\mathrm{THz}}[\mu \mathrm{J}]$ (Fig. 3). This dependence should be linear for a Gaussian beam. Thus, the threshold energy $W_{\text {th }}$ could be derived from the line's intersection with an X-axis, while the line's slope determines the parameter of the Gaussian beam at the level of $1 / e$.

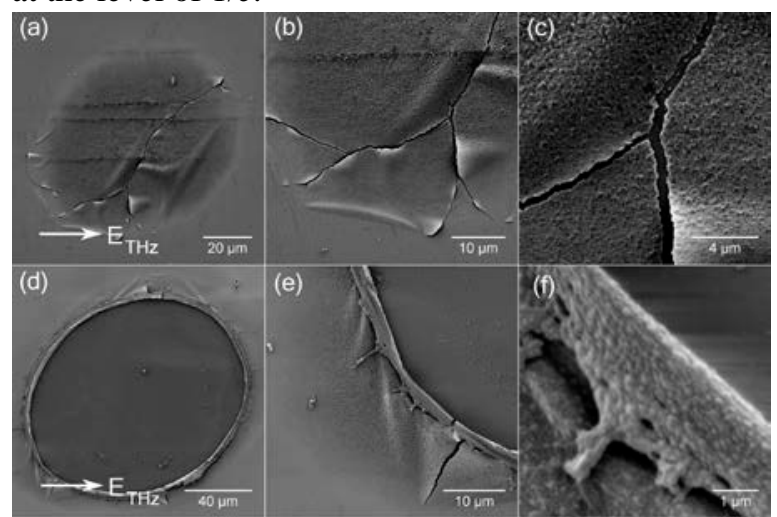

Fig. 1. SEM images of through holes and its edges in $\mathrm{Al}$ film produced by single $\mathrm{THz}$ pulses. (a), (b), (c): $F=0.14 \mathrm{~J} / \mathrm{cm}^{2}$; (d), (e), (f): $F=0.3 \mathrm{~J} / \mathrm{cm}^{2}$. The arrows indicate the direction of the $\mathrm{THz}$ electric field.
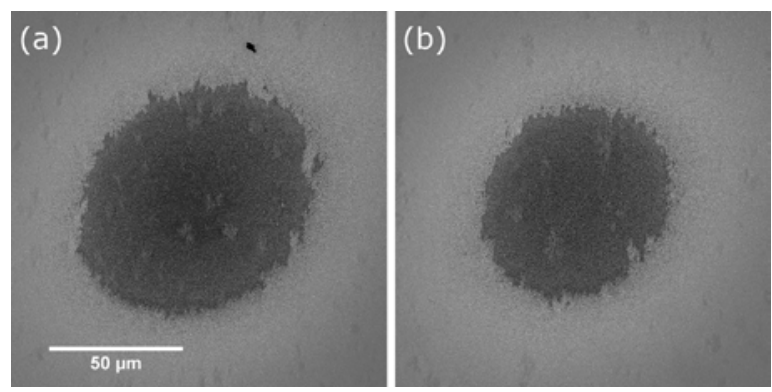

Fig. 2. SEM images of Ni film damages induced by single terahertz pulse at different fluences. (a): $F=0.43 \mathrm{~J} / \mathrm{cm}^{2}$, (b): $F=0.39 \mathrm{~J} / \mathrm{cm}^{2}$. The direction of the $\mathrm{THz}$ electric field is the same as in Fig. 1.

The experimental data in Fig. 3 are well approximated with a linear function (solid lines) indicating a Gaussian profile of the terahertz beam, high homogeneity of the used metal film and a distinct threshold behavior of its damage. The measured radius at the level of $1 / e$ was of $r_{0} \sim 90 \div 92 \mu \mathrm{m}$, which gives a single-pulse damage threshold of the incident fluence of $F_{\mathrm{th}}=W_{\mathrm{th}} /\left(\pi r_{0}^{2}\right) \approx 0.15 \mathrm{~J} / \mathrm{cm}^{2}$ for $\mathrm{Al}$ and $0.19 \mathrm{~J} / \mathrm{cm}^{2}$ for $\mathrm{Ni}$ at an electric-field strength about $15 \mathrm{MV} / \mathrm{cm}$ at the center of the focal spot. The value of electric-field strength was estimated using the energy of a $\mathrm{THz}$ pulse, 
its duration and a focused to a diffraction-limited spot size, assuming a Gaussian pulse shape [2].

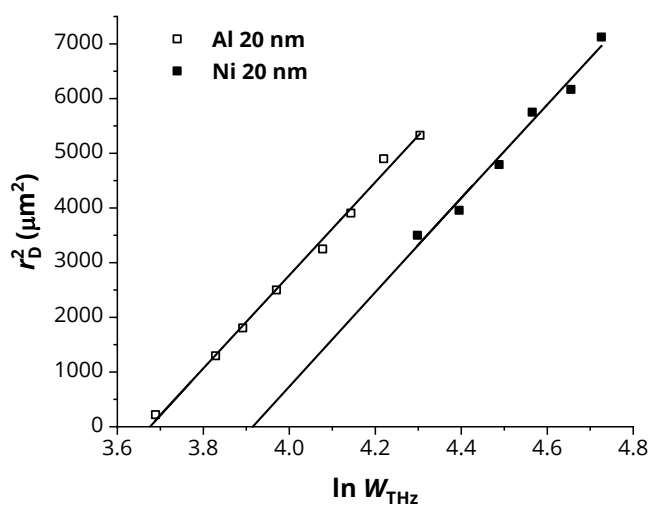

Fig. 4. Determination of the damage threshold in $\mathrm{Al}$ and $\mathrm{Ni}$ films irradiated with a single $\mathrm{THz}$ pulse; experimental data are shown with square symbols approximated with linear functions (solid lines).

SEM images of damage patterns in the $\mathrm{Al}$ and $\mathrm{Ni}$ films induced by multiple $\mathrm{THz}$ pulses at different fluences above the single-pulse damage threshold and number of pulses are shown in Fig. 4 and Fig. 5. The results were obtained at a pulse repetition rate of $10 \mathrm{~Hz}$.

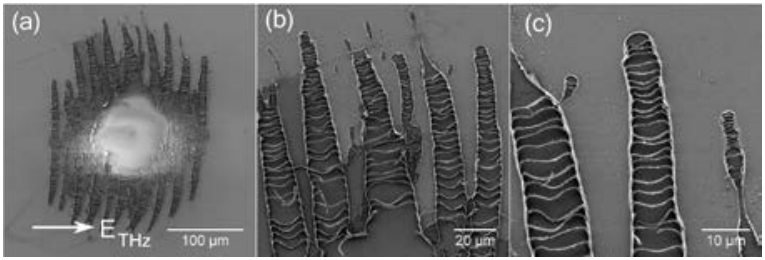

Fig. 4. SEM image of aluminum film damages induced by multiple THz pulses at fluence $F=0.24 \mathrm{~J} / \mathrm{cm}^{2}, N=60$.

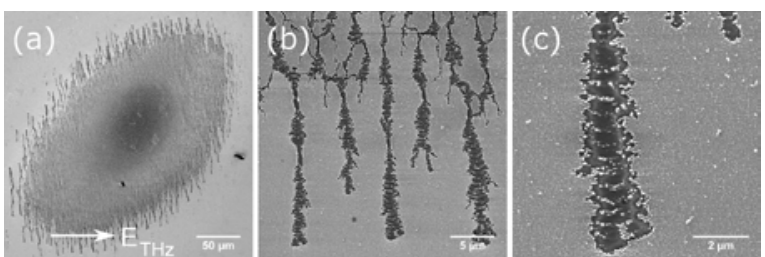

Fig. 5. SEM image of nickel film damage induced by multiple THz pulses at fluence $F=0.43 \mathrm{~J} / \mathrm{cm}^{2}, N=40$.

We suppose the observed single $\mathrm{THz}$ pulse-driven damages in thin metal films to be formed as a result of absorption of the $\mathrm{THz}$ pulse energy by conduction electrons with the following generation of hot electrons that transfer energy to the lattice and heat it. Depending on the fluence the lattice heating after the THz pulse could result in film damage due to thermal expansion or melting and ablation processes. Due to differences in acoustical impedances at film boundaries the film center of mass gains momentum in the direction from the substrate. Besides, a tensile stress wave results in adhesive debonding. Delamination of the film occurs when exceeding the adhesion strength. With increasing fluence of the $\mathrm{THz}$ pulse the film expands into free space and breaks down.

Damage behavior of metal films irradiated with multiple $\mathrm{THz}$ pulses at fluences below the single pulse damage threshold (at the periphery, where according to Gaussian distribution the fluence is below the singlepulse damage threshold) is rather challenging to explain. We suppose that the THz pulse at the center of the focused spot (a region with the peak electric field) generates a microcrack as a result of induced stress. It might be supposed that for the subsequent THz pulses there is a local field enhancement in the gap of the induced microcrack. This results in a microcrack growth perpendicular to the electric-field vector and an increase in width in across-track direction. The microcrack growth occurs along with film ablation in microregions similar to the mechanism of the single pulse damage. As for the Al film, the peripheral damage pattern has the form of elongated surface discontinuities (a kind of channels) that are perpendicular to the electric field vector of the terahertz pulse. However, these "channels" in the Ni film differ in character (pattern) and sizes. For the Al film the observed damages have clearly defined plastic behaviour (the damage surface is smooth, there are smooth and rolled up edges, a rim at the edge of damage is $1-2 \mu \mathrm{m}$ in width). For the $\mathrm{Ni}$ film the damage pattern is similar to fragile or quasi-fragile type (the damaged surface is rough and grainy, there are no smooth edges and rims). This could be associated with different physical and adhesion properties of the films (the Ni film has an increased adhesion as opposed to the Al film), and different processes of the film formation on a substrate during sputtering. Explain the difference in the size and periodicity of the structures formed on the surface of the films is not yet possible. A mechanism related to the generation of such channel-like damages and based on a new phenomenon of electrostriction in thin metal films was proposed in [2], while this phenomenon has not been and could not be observed in bulk metals due to a strong damping of the electric field.

This work is supported by Russian Science Foundation Grant No. 17-19-01261. The experiments were performed using the unique scientific facility "Terawatt Femtosecond Laser Complex" in the "Femtosecond Laser Complex" Center of the Joint Institute for High Temperatures of the Russian Academy of Sciences.

\section{References}

1. Agranat, M. B., Ashitkov, S. I., Ivanov, A. A., Konyashchenko, A. V., Ovchinnikov, A. V., Fortov, V. E. Terawatt femtosecond Cr:forsterite laser system // Quantum Electron. 2004. V. 34, No. 6. P. 506-508.

2. Agranat, M. B., Chefonov, O. V., Ovchinnikov, A. V., Ashitkov, S. I., Fortov, V. E., Kondratenko, P. S. Damage in a Thin Metal Film by High-Power Terahertz Radiation // Phys. Rev. Lett. 2018. V. 120, P. 085704.

3. Chefonov, O. V., Ovchinnikov, A. V., Evlashin, S. A., Agranat, M. B. Damage Threshold of Ni Thin Film by Terahertz Pulses // has been accepted for publication in Journal of Infrared, Millimeter, and Terahertz Waves. 